\title{
Reconsidering the Family History in Primary Care
}

\author{
Eugene C. Rich, MD, Wylie Burke, MD, PhD, Caryl J. Heaton, DO, Susanne Haga, PhD, \\ Linda Pinsky, MD, M. Priscilla Short, MD, Louise Acheson, MD, MS
}

OBJECTIVE: The purpose of this paper is to review the role of the family history in predictive genetic testing, describe how family history taking is practiced in adult primary care, identify the current barriers to appropriate application of the family history, and outline the requirements for a new family history tool for primary care.

DESIGN: We reviewed current perspectives on the family history, identifying key references in the medical literature and web-based family history tools through discussions with multiple content experts in clinical genetics, family medicine, and internal medicine. We conducted a Medline query using the search terms family history and primary care to identify references from the past 10 years. To illustrate the usefulness of family history information, we calculated the predictive value of family history and genetic information for familial adenomatous polyposis using current references and standard formulas. We identified paper and web-based family history tools through discussions with content experts. We also conducted a search on the World Wide Web to identify resources for electronic medical record and family history.

RESULTS: The family history is the most important tool for diagnosis and risk assessment in medical genetics, and promises to serve as a critical element in the use of predictive genetic testing in primary care. Traditional medical education about family history has often been unsophisticated and use of family history in adult primary care has been limited, compounded by multiple substantive barriers. Although there are numerous paper and computer-based aides for taking the family history, none currently meets all the needs of adult primary care.

CONCLUSIONS: The patient's family history remains a critical element in risk assessment for many conditions, but substantive barriers impede application in primary care practice, and evidence for its contribution to improved health outcomes

Received from the Department of Medicine (ECR), Creighton University, Omaha, Neb; Department of Medical History and Ethics (WB), University of Washington, Seattle, Wash; Department of Family Medicine (CJH), University of Medicine and Dentistry, New Jersey-Robert Wood Johnson Medical School, New Brunswick, NJ; The Center for the Advancement of Genomics (SH), Rockville, Md; Department of Medicine (LP), University of Washington, Seattle, Wash; Office of Biomedical Science and Clinical Research (MPS), American Medical Association, Chicago, Ill; and the Department of Family Medicine (LA), Case Western Reserve University, Cleveland, Ohio.

Address correspondence and reprint requests to Dr. Rich: Creighton University Department of Medicine, $601 \mathrm{~N}$. 30th Street, Ste.5850, Omaha, NE 68131 (e-mail: richec@creighton.edu). is limited in this setting. Short of radical changes in reimbursement, new tools will be required to aid primary care physicians in the efficient collection and application of patient family history in the era of genetic testing.

KEY WORDS: genetics; primary care; family history; genetic testing; medical history taking; pedigree.

J GEN INTERN MED 2004;19:273-280.

$T$ he Human Genome Project, with its many advances in the understanding of the genetic basis of human disease, has fostered hundreds of genetic tests and greatly heightened public awareness regarding the heritable basis of illness. As a result, the patient's family history is taking on renewed importance in adult primary care practice, as a means to assess risk and identify candidates for genetic testing or targeted medical care. In this paper, we review the family history as practiced by the current experts in heritable disease and genetic testing, medical genetics specialists. We also review the role of the family history in traditional primary care preventive practice as well as in the new and diverse applications of predictive genetic testing. We describe the family history as taught and practiced in adult primary care and review the current barriers to improving the documentation and use of the family history by primary care professionals. Finally, we review relevant tools and identify the potential characteristics of a new family history, which could bring adult primary care into the era of genetic medicine.

\section{Impending Implications of Genetics in Primary Care}

Advances in human genetics offer unprecedented opportunities for improved understanding and treatment of common illnesses. Already a wide range of relatively common adult conditions has been reported to have a genetic component. These include diabetes, Alzheimer's disease, venous thromboembolism, cardiovascular disease, congenital hearing loss, iron overload, schizophrenia, bipolar disease, depression, and numerous malignancies. ${ }^{1}$ Furthermore, genetic testing is available for a startling number of common and rare conditions. More than 540 genetic tests are presently available to clinicians and an additional 350 are currently under development. ${ }^{2}$ Francis Collins (Director of 
Table 1. Typical Information Obtained in Three-Generation Pedigree $^{6}$

Age or year of birth

Age and cause of death (for those deceased)

Ethnic background of each grandparent

Relevant health information (e.g., height and weight)

Illnesses and age at diagnosis

Information regarding prior genetic testing

Information regarding pregnancies including infertility,

spontaneous abortions, stillbirths, and pregnancy

complications

Information also obtained for half siblings

Consanguinity issues directly addressed

the National Human Genome Research Institute) recently predicted that the major contributing genes for heart disease, Alzheimer's, Parkinson's, and asthma will be identified in the next 5 to 7 years, ${ }^{3}$ observing in addition "virtually every human illness has a hereditary component." Medical care will be changed fundamentally as advances in understanding the genetic basis of disease become incorporated into diagnosis, treatment, and prevention.

\section{Medical Genetics and the Family History}

Medical geneticists and genetic counselors are the clinical experts in the use of genetic tests for the diagnosis and management of heritable diseases. Medical genetics professionals rely heavily on the family history as "the most traditional diagnostic tool in clinical genetics." ${ }^{5}$ Robin Bennett describes the family history as the "gateway to recognizing inherited disorders in a patient...." In genetics, the family history is defined as the description of the genetic relationships and medical history of a family; when the family history is represented in diagram form using standard symbols and terms, it is referred to as a pedigree. ${ }^{7}$

The traditional process of taking a family history in medical genetics involves several steps. A family medical history questionnaire is often the first step in this process and patients may be encouraged to contact relatives to obtain or confirm information. ${ }^{6,8}$ Many genetics professionals also conduct a preliminary telephone interview to assist the data collection process. The face-to-face interview is a critical part of eliciting a family history, with the ultimate goal to obtain a pedigree that includes at least 3 generations. The information geneticists typically record in a pedigree is listed in Table $1 .{ }^{6}$

The taking of the family history does not end with patient questionnaires and interviews, however. Numerous studies have demonstrated that patients' recall of their family history is frequently inaccurate. ${ }^{9-11}$ Many authorities in genetics emphasize that before physicians make testing or management decisions based on the patient's family history, effort should be made to confirm the reported illnesses (e.g., interviews of additional family members, review of medical records, autopsy reports, death certificates). ${ }^{6,8}$
Thus, substantial resources are devoted by the medical genetics team (medical geneticists and genetics counselors) to collect the family history, including considerable time to review medical family history questionnaires, conduct telephonic previsit patient interviews, interview family members, and locate and review family medical records. Bernhardt and Pyeritz surveyed genetics clinics and reported that 3 to 5.5 hours were spent per initial consultation, with over half of this time occurring before or after the actual clinic visit. ${ }^{12}$ The medical geneticist's effort to obtain the family history serves multiple purposes, including the establishment of rapport, the education of the patient and family about the genetic condition, and the facilitation of patient decision making. Despite the obvious importance of these benefits, the most important role of the family history is as a tool for genetic diagnosis and risk assessment. ${ }^{6}$

\section{The Family History as the Primary Predictive Genetic Test in Primary Care}

The family history has long been recognized as a risk assessment tool in primary care. ${ }^{13}$ As new genetic tests emerge, it has particular importance as a means of identifying people with increased risk for genetic disorders or susceptibilities. People at increased risk because of their family history may be among the most appropriate candidates for genetic tests. A traditional screening test is defined by both positive predictive value-the chance of having the disease under consideration if the test result is positive-and negative predictive value - the chance of not having the disease if the test is negative. Predictive value is a function of the test characteristics (sensitivity and specificity) and most importantly of the patient's "pretest probability" of disease, that is, the patient's personal risk of having the illness being screened. As shown in Table 2, if patients being tested have a low risk of the disease (e.g., $1 / 1,000)$, they are likely to be healthy even if their screening test is positive. Thus, the positive predictive value (chance that the screened individual actually has the disease) is only 9/100 when using an unusually accurate test with 99\% sensitivity and 99\% specificity (far better diagnostic accuracy than typically applicable to most screening tests in use today). In choosing screening tests, the background or population risk reflects the proportion of individuals in

Table 2. Prior Probability and Predictive Value

\begin{tabular}{lcr}
\hline \hline $\begin{array}{l}\text { Prior } \\
\text { Probability }\end{array}$ & $\begin{array}{c}\text { Positive Predictive } \\
\text { Value, } * \%\end{array}$ & $\begin{array}{r}\text { Positive Predictive } \\
\text { Value, }\end{array} \%$ \\
\hline $1 / 10000$ & $0.02 \%$ & $0.10 \%$ \\
$1 / 1000$ & $2.00 \%$ & $9.00 \%$ \\
$1 / 100$ & $16.00 \%$ & $50.00 \%$ \\
$1 / 10$ & $68.00 \%$ & $92.00 \%$ \\
$1 / 2$ & $95.00 \%$ & $99.00 \%$ \\
\hline
\end{tabular}

* 95\% sensitivity, 95\% specificity.

${ }^{\dagger}$ 99\% sensitivity, 99\% specificity. 


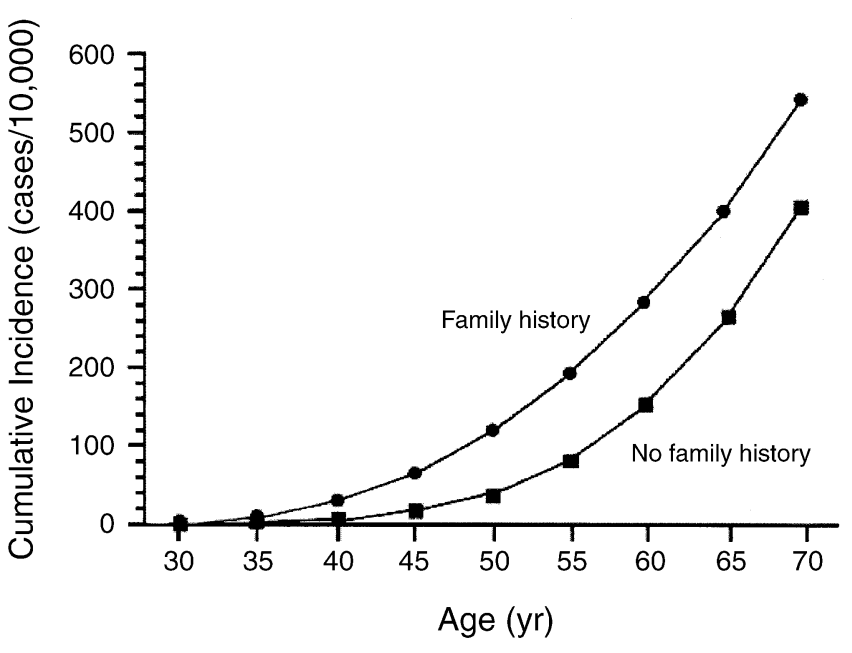

FIGURE 1. Colorectal CA risk \pm family history. From Fuchs et al. ${ }^{14}$

the general population affected with a particular disorder, ${ }^{7}$ but the patient's personal risk may be higher given his or her family history or other circumstances. Therefore, family history can be crucial information increasing the individual patient's pretest probability, and therefore the predictive value of genetic or other screening tests.

The role of family history in the predictive value for screening can be illustrated with colorectal cancer. The overall population risk is approximately $1 / 1,000$ for a 45year-old. Eight percent of the population have a first-degree relative with a history of colorectal cancer that confers a personal risk of colorectal cancer 2-fold higher than the general population $^{14}$ (Fig. 1), and individuals with a positive family history also present earlier with colorectal cancer. ${ }^{14}$ As a result, the estimated risk at age 40 is similar to that of an average risk person at age 50 (Fig. 1). Assuming that the sensitivity and specificity of the screening tests for colorectal cancer (e.g., fecal occult blood testing, colonoscopy) are the same for people in their 40s as for older patients, an argument can be made for initiating colorectal cancer screening at age 40 for patients with a positive family history. ${ }^{15,16}$ Thus, even before consideration of genetic testing, the family history has been important in determining the appropriate use of traditional screening tests.

Predictive use of genetic testing may be defined as testing offered to asymptomatic individuals in order to determine their risk to develop the disorder themselves. ${ }^{7}$ Determining the predictive value of these genetic tests involves the same mathematical principles as traditional screening tests. Note, however, that the predictive value of a genetic test may be subject to 2 different interpretations. It could be considered the likelihood that an individual with a positive result actually has the gene mutation in question. Alternatively, predictive value can be interpreted as the chance that the individual with a positive genetic test result will develop the associated disease. Medical geneticists address this dual meaning through the concepts of "analytic validity" and "clinical validity." Analytic validity refers to the accuracy with which the test identifies the mutation, whereas clinical validity refers to the accuracy with which the test result predicts clinical outcome (which is a function of gene penetrance). ${ }^{17}$ Given the incomplete penetrance of many mutations, the clinical validity of many genetic tests is lower than the analytic validity. ${ }^{17}$ These concepts apply to family history as well as to molecular genetic tests; thus, the analytic validity of family history as a risk predictor may be limited by the patient's knowledge about family members; and the clinical validity may depend on such factors as number of affected relatives and age of onset of disease. For many diseases, there are insufficient data to provide precise estimate of the analytic and clinical validity of a positive family history, although it is a well-established risk factor for most common diseases. ${ }^{18}$

Family history plays an important role in identifying patients who may benefit from predictive genetic testing. Familial adenomatous polyposis (FAP), in which innumerable colon polyps develop at an early age, offers an excellent example. The population or background risk of FAP is 1/ 8,000; individuals who inherit a mutation in the APC gene causing FAP are estimated to have a risk of colorectal cancer that approaches $100 \%$ (see Table 3). Conversely, the background population's lifetime risk of colorectal cancer is 5/100. A positive family history of FAP dramatically influences the patient's personal lifetime risk of colorectal

Table 3. Genetic Testing for FAP

\begin{tabular}{llcc}
\hline \hline & $\begin{array}{c}\text { General } \\
\text { Population }\end{array}$ & $\begin{array}{c}\text { Affected First } \\
\text { Degree Relative }\end{array}$ & $\begin{array}{c}\text { Affected First Degree } \\
\text { Relative With Identified Mutation* }\end{array}$ \\
\hline Risk for FAP in tested individual & $1 / 8000$ & $1 / 2$ & $1 / 2$ \\
Test sensitivity, \% & 95 & 95 & 99.9 \\
Test specificity, \% & 99.9 & 99.9 & 99.9 \\
Positive predictive value, \% & 10.61 & 99.89 & 99.99 \\
Negative predictive value, \% & 0.00006 & 4.77 & 0.10 \\
\hline
\end{tabular}

* In practice, medical geneticists seek to increase the predictive value of many genetic tests further by a testing protocol in which a family member with clinically established disease (e.g., FAP) is tested first. If this test identifies a specific causative mutation, a more sensitive predictive test is then offered to unaffected family members.

FAP, familial adenomatous polyposis. 
cancer. If a parent has FAP, the risk of the individual patient having an APC mutation associated with FAP is $50 \%$. Penetrance is estimated to approach $100 \%$, so lifetime risk is close to $50 \%$. Current sensitivity of full gene sequencing analysis for predicting FAP is approximately 95\%. Taken together, these facts suggest that predictive testing for FAP can identify individuals with this conditionthat is, can provide a genetic diagnosis of FAP. Preventive treatment, in the form of a subtotal colectomy, can be offered to reduce the risk of developing colorectal cancer.

This predictive testing scenario must, however, take into account limitations in test specificity. Because of the precision of molecular testing, the analytic validity of genetic tests is estimated to be very high, although for most genetic conditions, the general population studies have not been performed to determine the actual rate of false positive test results (i.e., specificity). If we assume, for the purpose of this example, that the accumulated random or procedural errors related to genetic testing for FAP yield a potential specificity of $99.9 \%$ (i.e., a false positive rate of $1 / 1,000)$, genetic testing for FAP in the baseline population (where risk of FAP is $1 / 8,000$ ) would result in a positive predictive value of $11 \%$ (Table 3 ). That is, despite the high sensitivity and specificity of FAP testing, and the high penetrance of FAP mutations, most of the positive test results occurring with FAP testing of the general population would represent false positives. In contrast, if the same genetic test were conducted on a patient with a positive family history of FAP, the predictive value of the positive test (i.e., the patient's risk of contracting FAP-related colorectal cancer) would be $99.9 \%$ (Table 3). Therefore, the individual patient's family history remains a critical factor in determining the value of predictive genetic testing.

\section{The State of the Family History in Adult Primary Care}

Is the family history, as currently practiced in adult primary care, sufficient for the appropriate selection and interpretation of genetic tests?

One can find traditional 20th century medical student education about the family history in DeGowin and DeGowin's classic, "Bedside Diagnostic Examination." 19 In this text, obtaining the family history involves documenting parents' and siblings' age and health (or age and cause of death), as well as a checklist of conditions with environmental as well as hereditary etiologies: "exposure to tuberculosis, syphilis, leprosy. History of hypertension, heart disease, kidney disease, arthritis...mental or emotional disturbances, alcoholism, epilepsy." ${ }^{19}$ While one current general medicine text emphasizes that "the key to determining the inherited risk for common adult onset diseases still rests in the collection and interpretation of a detailed personal and family medical history in conjunction with the directed physical examination," ${ }^{20}$ current physical diagnosis texts remain inconsistent and incomplete regarding the conditions and approach that should be the focus of a genetically informed family history. ${ }^{21,22}$
Family medicine has long emphasized that the primary focus of medical care is the patient in the context of the family. ${ }^{23}$ Rakel's "Textbook of Family Practice" has critiqued traditional medicine's "checklist" approach to the family history: "too often...this ritualistic inquiry...[is] no more than a recitation... of diseases...for which a yes or no answer is requested." ${ }^{24}$ Recognizing explicitly the time and effort involved in obtaining a thorough family history, Rakel suggests "the family physician usually accumulates a complete family history over a period of time during a series of patient visits." ${ }^{24}$ Rakel further recommends that this family history information be presented graphically in a special form of pedigree termed a "genogram."

A genogram graphically presents the biological, social, and psychosocial issues in a multigenerational family history. ${ }^{25,26}$ The genogram grew out of the social work and family therapy disciplines; as it has evolved in family medicine, the genogram has become a "pictorial display of the patient's family relationships and medical history," collecting and recording 3 generations of family information in a variety of categories. Traditional pedigree information is presented, but, in addition, the genogram depicts important relationship information such as family members living in the same household, and relationship patterns (for example, close vs cut-off vs conflicted relationships). Functional charting of social and interpersonal relationships is a unique feature of genograms. ${ }^{25}$ An example of a genogram may be viewed at the following website: http:// www.aafp.org/fpm/20010300/genogram.pdf. Accessed January 21, 2004.

Despite the longstanding emphasis on collecting family history data in family medicine, and the more recent emphasis on taking a genetically informed family history in internal medicine, evidence from practice suggests that a thorough exploration of the family history may be more the exception than the rule in adult primary care. In 1986, Crouch and Thiedke audited randomly selected charts in a university-based family practice residency, finding that family histories of alcoholism, emphysema, and mental illness were seldom recorded. ${ }^{27}$ In a survey by Acton et al., $63 \%$ of family physicians, $74 \%$ of internal medicine physicians, and $85 \%$ of obstetrician/gynecology physicians reported obtaining family histories on at least three quarters of their patients. ${ }^{28}$ A survey of 1,642 physicians in the Pacific Northwest provided more encouraging results, finding that $95 \%$ of physicians reported taking a family history. ${ }^{29}$ However, other data suggest that the quality and thoroughness of these family histories might be lacking.

The Direct Observation of Primary Care study has provided considerable additional information on the use of the family history in family medicine. This study involved observation of 4,054 outpatient visits for 138 community physicians in 84 practices. This work has documented, for example, that family histories were discussed during $51 \%$ of new patient visits and $22 \%$ of follow-up visits. Genograms were present on only $11 \%$ of patient charts. ${ }^{30}$ Further analysis has demonstrated a substantial physician- 
specific variation in the rate of obtaining family history information; some family physicians obtain family histories rarely while others obtain family histories on $80 \%$ of visits. $^{31}$ Not surprisingly, there is a higher rate of family history taking by younger family physicians, but the length of family history discussions is startlingly brief compared to medical geneticists'-less than 3 minutes for new patients and less than 2 minutes on return visits. ${ }^{31}$ This may explain why genograms are not commonly found in the medical records of these family physicians. In 1984, Rogers and Durkin estimated that initial construction of a 3-generation genogram took 15 to 20 minutes, and Waters subsequently reported that a more complete 3-generation genogram takes perhaps 30 minutes. ${ }^{32,33}$

\section{Barriers to Family History Taking in Primary Care}

Thus, despite recent teaching on the importance of the family history in primary care, there appear to be significant barriers to obtaining family history information in primary care practice. Acton et al. found that primary care physicians were likely to note "lack of time during visit" as a barrier to genetic counseling. ${ }^{28}$ Certainly the time devoted by primary care physicians to obtaining family history information is typically far less than the time suggested by experts in family medicine and internal medicine, and strikingly less than that invested by medical genetics professionals.

Current reimbursement policies for primary care may complicate the ability of adult primary care physicians to devote the time and infrastructure to obtaining adequate family histories. In one recent analysis using current guidelines, ${ }^{34,35}$ taking a family history would increase reimbursement for only 1 new patient Evaluation and Management CPT code, 99202, and 1 return visit code, 99213; this action would only increase reimbursement enough to support approximately 10 minutes of physician effort for collecting, documenting, and analyzing the family history. ${ }^{35-37}$ Of course, physicians provide many services for patients for which they are not directly reimbursed. ${ }^{38}$ Primary care physicians are increasingly practicing in group practices that are struggling with rising overhead and stagnant or declining reimbursements, however. In these settings, primary care physicians may not be encouraged by their physician partners, nor by their busy patients, ${ }^{39-41}$ to devote the time, energy, and infrastructure necessary for the exhaustive collection and interpretation of family history information.

Primary care physicians may also be discouraged by their perceived limited knowledge and skills regarding advances in human genetics. Several studies in Great Britain have shown many general practice physicians do not feel they have the skills to take an adequate family history and counsel patients on cancer risk. ${ }^{42,43}$ Similarly, Australian general practitioners report their knowledge of genetics to be poor and perceive themselves to be inadequately prepared to manage patients with genetic conditions. ${ }^{44}$ Even in a highly selected group of general internal medicine, general pediatrics, and family practice clinician educators (participants in the Genetics in Primary Care Faculty Development Initiative), only half reported having sufficient genetics knowledge to incorporate recent advances into their primary care practice. ${ }^{45}$

Thus, there are substantial barriers to enhancing family history taking in primary care, including reimbursement policies for primary care physicians, current modes of organizing adult primary care practices, varying patient expectations, and the physician's own knowledge and skills. Physician knowledge can be successfully addressed through educational programs, as demonstrated by the success of initiatives like the Genetics in Primary Care (GPC) program. ${ }^{45}$ Overcoming the problem of the time and effort required for collection and analysis of the family history remains a daunting challenge, however.

\section{Family History Tools Applicable to Primary Care}

Short of radical changes in reimbursement of primary care practice, new tools will be required to aid primary care physicians in the efficient collection and application of patients' family history in the era of genetic testing. Various paper family history tools are available. ${ }^{46}$ As noted earlier, medical genetics professionals and family physicians ${ }^{31}$ often use family history forms as an aid to the overall preparation of the family history. In addition, there are various computer-based tools for drawing pedigrees (e.g., Progeny, Genosketch, and others), ${ }^{6}$ and family history documentation tools can be downloaded from the internet. ${ }^{46-48}$ However, having genetics professionals construct the pedigree in digital form, using such tools, does not appear to reduce the estimated 30 to 90 minutes required to collect genogram and pedigree data. ${ }^{49}$

Electronic medical records are increasingly integrated into medical practice and numerous examples are available for review on the internet. ${ }^{50}$ While some of these represent merely an "electronic chart," providing a location titled Family History for traditional "free text" documentation, others allow the development of family history templates, supporting the longstanding "checklist" approach to taking and documenting the family history. We have found no electronic medical record systems that display the family history graphically as a pedigree or genogram. Thus, most current electronic medical record products do not represent a dramatic enhancement to traditional family history-taking strategies.

Currently available tools thus appear to offer little help overcoming the barriers to competent family history applications in primary care. For these reasons, the primary care physician educators involved in the GPC project have emphasized the need for new resources to support the incorporation of genetic advances into primary care practice. A GPC Project workgroup has identified the characteristics of the ideal family history tool (Table 4). In this group's conception, the patient could perform the family medical history collection and input before seeing their 
Table 4. The Ideal Family History Tool

Patient-completed (e.g., paper, desktop, telephone, or web input) Adapted to patient age, gender, ethnicity, common conditions Elicits specific patient concerns Brief, understandable, easy to use

Compatible with multiple clinical applications (paper, EMR, PDA) Contains clinical decision support

Branches and prioritizes based on clinical significance

EMR, electronic medical record; PDA, personal digital assistants.

physician. They emphasize that the ideal tool would need to be modified for patient age, gender, ethnicity, and health condition. In primary care, in contrast to genetic consultation, the family history could appropriately begin with "triage" or screening questions, positive answers to which would lead to more detailed family history taking when appropriate to the clinical situation. To be helpful to patients, the tool must be understandable (fitting the patient's language and educational level) and easy to use. It must also be adaptable to multiple media (including paper and internet input). Given the variations in office information systems and record keeping, this family history tool should be compatible with multiple clinical information system applications. Web-based and telephone-based tools for patient entry of genetic risk information are in development, with variable degrees of portability and download ability into physician records. ${ }^{49}$ While the "portable electronic family history" is not yet a reality, clearly the technologic capacity is at hand.

Equally important would be a clinical decision support function relevant to primary care, to speed accurate interpretation and effective application of family history information. ${ }^{51}$ Paper versions of this kind of clinical decision support function are already represented in certain patient-completed forms. ${ }^{52}$ Expansion of this approach could include electronic technology ${ }^{51}$ and a wider array of conditions relevant to the evaluation of family history in internal medicine and family practice.

The application of personal digital assistants (PDA) to "point of care computing" represents another potential revolution that might ensure the effective and appropriate application of electronic family history information in primary care practice. For several years, prediction rules relevant to genetic testing (such as the Gail model for breast cancer risk) have been available through PDA software such as MedRules. ${ }^{53}$ These are very specific and problemfocused utilities, providing nothing in the way of recommended actions or advice. More sophisticated applications of point-of-care computing technology are now available, however. For example, the complex cholesterol management guidelines developed in Adult Treatment Panel III (ATP III) are now downloadable into an "Interactive Guideline Tool." ${ }^{54}$ This tool allows input of patient-specific information, including family history, and then presents recommended actions based on the patient's specific constellation of risk factors. The Interactive Guideline Tool also provides access to the rationale and evidence base for these recommendations. Advances in the processing speed and memory capacity of PDAs, as well as the development and impending dissemination of new generations of PDAs with wireless internet connection, provide an exciting opportunity for overcoming current barriers in the application of family history information in primary care. Much work remains to be done regarding the development of the patient-derived electronic family history database, as well as guidelines and software for primary care practice applications. Nonetheless, one can envision a future where portable electronic family history data integrated through electronic medical records to PDAs and evidence-based practice guidelines could overcome current barriers to the thorough collection, accurate interpretation, and wise application of the family history in primary care practice.

Unfortunately, such tools are years in the future, but primary care physicians must wisely collect and use family history information today. Based on reviews of the medical literature and textbooks, there is presently no consensus on the current best approach to taking the family history in adult primary care. While the approach taken by subspecialists in medical genetics may be viewed as the "gold standard" in authoritative texts, ${ }^{20}$ evidence is lacking regarding the cost-effectiveness of this approach in general medical care. Work is ongoing to evaluate new approaches to "screening" questions about family history that can be used to guide primary care teaching and practice. The Centers for Disease Control has recently launched an exciting project to develop a patient-completed family history tool relevant to health promotion in adults, the Family History Public Health Initiative. Until these efforts are completed, primary care physicians must carefully weigh the potential benefits of the family history information obtained with the costs and risks of obtaining it. As with all other case-finding data collection, ${ }^{13}$ this judgment will likely vary with the physician's patient population and practice circumstance, as well as emerging evidence regarding the predictive value of genetic tests for common diseases and benefits of riskbased prevention. Most of the authors find organizing family history information graphically in a pedigree or genogram format most helpful. Some have had success with family history questionnaires as well.

\section{CONCLUSION}

Advances in our understanding of the biology of human genetics have been startling and pose tremendous potential for benefits to clinical practice. These advances also pose enormous challenges and considerable risk of harm to patients. These risks include costly and unnecessary testing, needless anxiety, and inappropriate and possibly hazardous therapies. Furthermore, as medical genetics professionals well know, genetic testing can significantly affect family relationships, lifestyle choices, and reproductive decision making. While genetics professionals are well trained to apply family history information and 
genetic test results wisely to further individual and family well being, the number of these professionals is woefully lacking. Compounding this problem is the ubiquitous applicability of advances in human genetics. "All patients have genes"; therefore, an increasing proportion of clinical decisions will require the integration of family history and personal genetic risk information, not only for screening and diagnosis, but therapy as well. Clearly, 20th century teaching and technology for the family history will be inadequate to ensure that patients derive the full benefits from advances in human genetics. Radical new resources and approaches to the application of family history will be required in the coming years.

This work has been supported in part through funding from the Genetics in Primary Care (GPC): A Faculty Development Initiative; Michele Puryear, MD PhD, Project Officer. The GPC is funded by a contract from the Maternal and Child Health Bureau, Bureau of Health Professions, Health Resources and Services Administration (HRSA contract 240-98-0020) to the Society of Teachers of Family Medicine, with cofunding from the National Human Genome Research Institute, National Institutes of Health and the Agency for Healthcare Research and Quality.

The views and opinions expressed in this article are those of the authors and do not necessarily reflect those of the funding agencies involved.

The authors would like to acknowledge the excellent work of Teri Hoppe, Director of Operations for Creighton Medical Associates, and Christina Acosta, Compliance Coordinator, Creighton University, for the Coding Evaluation and Management Services analysis; and Beverly Repouille, Administrative Assistant, and Wendy Taylor, Senior Specialist, Creighton University, who provided invaluable editorial assistance in compiling this multiauthored work.

\section{REFERENCES}

1. Evans JP, Skrzynia C, Burke W. The complexities of predictive genetic testing. BMJ. 2001;322:1052-6.

2. GeneTests Website. Available at: http://www.genetests.org. Accessed May 22, 2002.

3. Collins FS, for the National Coalition for Health Professional Education in Genetics (NCHPEG). Annual Meeting. Genetics, Health Care, and NCHPEG. The Future Is Now. NCHPEG Annual Meeting, February 2002, Bethesda, Md.

4. Collins FS, McKusick VA. Implications of the Human Genome Project for Medical Science. JAMA. 2001;285:540-4.

5. Motulsky AG. Forward In: Bennett RL. The Practical Guide to the Genetic Family History. New York, NY: Wiley-Liss; 1999.

6. Bennett RL. The Practical Guide to the Genetic Family History. New York, NY: Wiley-Liss; 1999

7. GeneTests Website. Glossary. Available at: http://www.genetests.org. Accessed May 22, 2002.

8. Harrison's Online. Available at: http://www.harrisonsonline.com/. Accessed May 24, 2002.

9. Kerber RA, Slattery ML. Comparison of self-reported and databaselinked family history of cancer data in a case-control study. Am J Epidemiol. 1997;146:244-8.

10. King TM, Tong L, Pack RJ, et al. Accuracy of family history of cancer as reported by men with prostate cancer. Urology. 2002;59:546-50.
11. Hunt K, Emslie C, Watt G. Lay constructions of a family history of heart disease: potential for misunderstandings in the clinical encounter? Lancet. 2001;357:1168-71.

12. Bernhardt BA, Pyeritz RE. The economics of clinical genetics services. III. Cognitive genetics services are not self-supporting. Am J Hum Genet. 1989;44:288-93.

13. U.S. Preventive Services Task Force. Guide to Clinical Preventive Services, 2nd edn. Baltimore, Md: Williams \& Wilkins; 1996.

14. Fuchs CS, Giovannucci EL, Colditz GA, et al. A prospective study of family history and the risk of colorectal cancer. N Engl J Med. 1994;331:1669-74.

15. Winawer SJ, Fletcher RH, Miller L, et al. Colorectal cancer screening: clinical guidelines and rationale. Gastroenterology. 1997;112:594-642.

16. American Cancer Society Website. ACS Guidelines for Colorectal Cancer Screening Available at: http://www.cancer.org/docroot/ NWS/content/NWS_1_1x-asp.ACS_GUIDELINES_FOR_COLORECTAL CANCER_SCREENING. Accessed August 15, 2002.

17. Holtzman NA, Watson MS. Promoting safe and effective genetic testing in the United States. Final report of the task force on genetic testing. Baltimore, Md: Johns Hopkins Press; 1999.

18. Yoon PW, Scheuner MT, Peterson-Oehlke KL, et al. Can family history be used as a tool for public health and preventive medicine. Genet Med. 2002;4:3004-310.

19. Degowin EL, Degowin RL. Bedside Diagnostic Examination, 2nd edn. New York, NY: Macmillan Co; 1969.

20. Bruanwald E, Fauci AS, Kasper DL, et al., eds. Harrison's Principles of Internal Medicine, 15th edn. New York, NY: McGraw-Hill; 2001

21. Bickley LS, Hoekelman RA. Physical Examination and History Taking, 7th edn. Philadelphia, Pa: Lippincott, Williams \& Wilkins; 1999.

22. Swartz MH. Textbook of Physical Diagnosis: History and Examination, 2nd edn. Philadelphia, Pa: W.B. Saunders Co.; 1999.

23. Saultz JW, ed. Textbook of Family Medicine. New York, NY McGraw-Hill; 2000.

24. Rakel RE, ed. Chapter 2 in: Textbook of Family Practice. Philadelphia, Pa: Saunders WB Co.; 1990.

25. McGoldrick M, Gerson R, Shellenberger S. Genograms: Assessment and Intervention, 2nd edn. New York, NY: W.W. Norton; 1999:169-70.

26. Daly M, Farmer J, Harrop-Stein C, et al. Exploring family relationships in cancer risk counseling using the genogram. Cancer Epidemiol Biomarkers Prev. 1999;8:393-8.

27. Crouch MA, Thiedke CC. Documentation of family health history in the outpatient medical record. J Fam Pract. 1986;22:169-74.

28. Acton RT, Burst NM, Casebeer L, et al. Knowledge, attitudes, and behaviors of Alabama's primary care physicians regarding cancer genetics. Acad Med. 2000;75:850-2.

29. Hayflick SJ, Eiff MP, Carpenter L, et al. Primary care physicians' utilization and perceptions of genetics services. Genet Med. 1998;1: 13-21.

30. Medalie JH, Zyzanski SJ, Goodwin MA, et al. Two physician styles of focusing on the family. J Fam Pract. 2000;49:209-15.

31. Acheson LS, Wiesner GL, Zyzanski SJ, et al. Family history taking in community family practice: implications for genetic screening. Genet Med. 2000;2:180-5.

32. Rogers J, Durkin M. The semi-structured genogram interview. I. Protocol, II. Eval Fam Sys Med. 1984;2:176-87.

33. Waters I, Watson W, Wetzel W. Genograms. Practical tools for family physicians. Can Fam Physician. 1994;40:282-7.

34. Health Care Financing Administration (HCFA) Documentation Guidelines; 1997. Available at. http://aaciweb.com/downloadfiles/ emguide.pdf. Accessed May 31, 2002.

35. Hoppe T, Acosta T. Internal report. Role of Family History in Coding Evaluation and Management Services, 2002. Department of Medicine. Creighton University, Omaha, Neb.

36. Part B News Group. E/M Bell Curve Data Book. Rockville, Md: UCG; 1999.

37. Medical Group Management Association, 1999. Compensation Production Survey. Englewood, Co: Medical Group Management Association; 2001. 
38. Bernhardt BA, Weiner J, Foster EC, et al. The economics of clinical genetics services. II. A time analysis of a medical genetics clinic. Am J Hum Genet. 1987;41:559-65.

39. Kravitz RL, Callahan EJ, Paterniti D, et al. Prevalence and sources of patients' unmet expectations for care. Ann Int Med. 1996; 125:730-7.

40. Rich EC. Whatever happened to managed care? Creighton Magazine. Winter, 2001:36-41.

41. Audrain J, Rimer B, Cella D, et al. Genetic counseling and testing for breast-ovarian cancer susceptibility: What do women want? J Clin Oncol. 1998;16:133-8.

42. Watson EK, Shickle D, Qureshi N, et al. The 'new genetics' and primary care: GPs' views on their role and their educational needs. Fam Pract. 1999;16:420-5.

43. Fry A, Campbell H, Gudmunsdottir H, et al. GPs' views on their role in cancer genetics services and current practice. Fam Pract. 1999; 16:468-74.

44. Metcalfe S, Hurworth R, Newstead J, et al. Needs assessment study of genetics education for general practitioners in Australia. Genet Med. 2002;4:71-7.

45. Bragg D, Simpson D, Treat R, Holloway R. The Genetics in Primary Care (GPC) Faculty Development Initiative Training Program: Final Evaluation Report. Submitted by the GPC External Evaluation Team from the Medical College of Wisconsin (Richard Holloway, PhD, Project Director) to the GPC Executive Committee; October 4, 2002.

46. American Medical Association's Adult Family History Form. Available at: http://www.ama-assn.org/ama/pub/article/ 2380-2844.html. Accessed May 22, 2002.
47. Oehlke KP. Documenting the Family Health History. An Overview of Available Tools. Presented at a workshop sponsored by Centers for Disease Control, Office of Genomics and Disease Prevention. Family History as a Tool for Public Health and Preventive Medicine. Decatur, Ga; May 1, 2002.

48. American Academy of Family Physicians Website. Example of Blank Genogram. Available at: http://www.aafp.org/fpm/20010300/ genogram.pdf. Accessed May 22, 2002.

49. Acheson LS, Stange KC, Zyzanski SJ, et al. A New Tool for Automating Family History Collection. Presented at the North American Primary Care Research Group Annual Meeting, Nova Scotia, Canada; November 15, 2001.

50. Physician Micro Systems, Inc(PMSI)Website Practice Partner Patient Records. Available at: http://www.pmsi.com/products/ patient_records/index.html. Accessed May 23, 2002.

51. Emery J, Walton R, Murphy M, et al. Computer support for interpreting family histories of breast and ovarian cancer in primary care: comparative study with simulated cases. BMJ. 2000;321:28-32.

52. The Programme: Primary Ob Genetic Risk Assessment and Management in MainE. Genetic Risk Screening Office Guide. Scarborough, Me: Foundation for Blood Research; 1996.

53. MedRules Website. Available at: http://pbrain.hypermart.net/ medrules.html. Accessed May 22, 2002.

54. National Cholesterol Education Program Website, Adult Treatment Panel III (ATP III) Interactive Guideline Tool. Available at: http:// hin.nhlbi.nih.gov/atpiii/atp3palm.htm. Accessed May 24, 2002. 